\title{
Multi-scale fracture of random heterogeneous materials
}

\author{
S. Rahman* \\ College of Engineering, The University of Iowa, Iowa City, IA, USA \\ (Received 28 May 2009; final version received 22 June 2009)
}

\begin{abstract}
This article presents new probabilistic models for generating microstructures and multi-scale fracture analysis of a random heterogeneous material. The microstructure model involves a level-cut, inhomogeneous, filtered Poisson field comprising a sum of deterministic kernel functions that are scaled by random variables and centred at Poisson points. The fracture model involves stochastic description of the particle volume fraction and locations and constituent material properties, two-scale algorithms including micro-scale and macro-scale analyses, and dimensional decomposition or Monte Carlo simulation for reliability analysis. Numerical results demonstrate that the random field model is capable of producing a wide variety of twoand three-dimensional microstructures containing particles of various sizes, shapes, densities, gradations and orientations. The results of fracture analysis indicate that the concurrent model developed is sufficiently accurate, gives probabilistic solutions very close to those generated from the micro-scale model and can reduce the computational effort of the latter model by more than a factor of two. In addition, the concurrent multi-scale model predicts crack trajectory as accurately as the micro-scale model. The stochastic models presented have the potential to fundamentally change the way advanced materials in high-technology applications, including the maritime industry, can be applied in the future.
\end{abstract}

Keywords: microstructure; filtered Poisson field; reliability; functionally graded material

\section{Nomenclature}

$\Omega, \mathcal{F}, P \quad=$ probability space, $\sigma$-field, measure

$\mathbb{R}^{d} \quad=d$-dimensional real vector space

$\mathcal{D}, \overline{\mathcal{D}} \quad=$ domain and subdomain

$\mathcal{D}_{p}, \mathcal{D}_{m} \quad=$ subdomains of particle and matrix

$\mathcal{N}^{\prime} \quad=$ Poisson random variable

$\lambda(\boldsymbol{x}) \quad=$ intensity function

$\boldsymbol{\Gamma}_{i}, \boldsymbol{\Phi}_{i} \quad=$ Poisson point and rotation matrix

$Z_{i} \quad=$ random scaling variable

$h \quad=$ kernel function

$Z(x) \quad=$ filtered Poisson field

$Y(x) \quad=$ level-cut filtered Poisson field

$I(z ; a), a \quad=$ indicator function and level

$p_{1} \quad=$ volume fraction

$p_{11}=$ two-point correlation function

$E_{p}, E_{m} \quad=$ elastic moduli of particle and matrix

$v_{p}, v_{m}=$ Poisson's ratios of particle and matrix

$\boldsymbol{C}(\boldsymbol{x}) \quad=$ elasticity tensor at point $\boldsymbol{x}$

$\boldsymbol{C}^{(p)}, \boldsymbol{C}^{(m)}=$ elasticity tensors of particle and matrix

$\overline{\boldsymbol{C}}(\boldsymbol{x}) \quad=$ effective elasticity tensor

$\boldsymbol{u}, \boldsymbol{\sigma}, \boldsymbol{\varepsilon}=$ displacement, stress, and strain fields

$\boldsymbol{R}, N=$ input random vector and its dimension

$f_{R}(\boldsymbol{r}) \quad=$ joint probability density of $\boldsymbol{R}$

$K_{I}, K_{I I} \quad=$ modes-I and -II stress-intensity factors

$K_{I c} \quad=$ mode-I fracture toughness

$y(\boldsymbol{R}) \quad=$ function for fracture initiation
$P_{F}\left(K_{I c}\right) \quad=$ conditional failure probability

$\tilde{M}^{(1,2)} \quad=$ interaction integral (inhomogeneous)

$M^{(1,2)} \quad=$ interaction integral (homogeneous)

\section{Introduction}

Research in heterogeneous materials has a long and revered history, involving the work of luminaries such as Maxwell (1873), Rayleigh (1892) and Einstein (1906), and has important ramifications in numerous fields, including engineering, material science, rheology, geophysics, statistical physics, chemical physics, colloid science, oil and gas exploration, and biology (Torquato 2002). An important class of heterogeneous media comprises composite materials, often manufactured by combining two or more constituent materials with significantly different physical, mechanical or chemical properties. In the maritime industry, an engineered composite material may exhibit superior fatigue and fracture characteristics than traditional materials (e.g. steel or aluminium) used in ships and offshore structures (Gillespie 1991). Additionally, compared with the traditional materials, composites have lower thermal conductivity, lighter weight, higher strength or stiffness and higher resistance to corrosion (Dutta 1994). However, the extent to which a composite material can be tailored to produce target mechanical performance depends on a sound theoretical

\footnotetext{
*Email: rahman@engineering.uiowa.edu
} 
understanding of the fracture behaviour and reliability of heterogeneous materials.

Many natural and man-made heterogeneous materials have a random internal structure comprised of two or more phases. There are two challenging problems in the study of a heterogeneous material: (1) modelling the microstructure of the material (Quintanilla and Torquato 1997; Roberts 1997; Grigoriu 2003; Rahman 2008); and (2) predicting the effective properties, such as transport, thermal, mechanical properties (Ferrante and Graham-Brady 2005; Rahman and Chakraborty 2007), including fracture performance (Chakraborty and Rahman 2008), from this microstructural information. Although research in solving these problems has exploded in recent years, fundamental progress in this field has been slow, if not hampered, due to, first, a lack of rigorous mathematical models capable of characterising complex microstructures. Second, most heterogeneous materials have multi-scale features; calculating their effective properties from rules of mixture, variational bounds or classical micromechanics can lead to inadequate or misleading measures of fracture performance. Therefore, new mathematical models that can accurately represent random microstructure and new multi-scale simulations that can render computationally expedient yet sufficiently accurate prediction of fracture response and reliability of a generic heterogeneous material are highly desirable.

This paper presents new stochastic models for random microstructure and multi-scale fracture analysis of heterogeneous materials. The microstructure model is based on a level-cut, inhomogeneous, filtered Poisson field comprising a sum of deterministic kernel functions that are scaled by random variables and centred at Poisson points. The fracture model involves stochastic description of the particle volume fraction, particle locations and constituent material properties; a two-scale algorithm including micro-scale and macro-scale analyses for determining crack-driving forces; and Monte Carlo simulation for reliability analysis. Section 2 presents the microstructure model, including an algorithm for generating samples of synthetic microstructures. Section 3 describes a fracture problem in functionally graded materials (FGMs), defines random input parameters and discusses crack-driving forces and reliability. Several multi-scale and micro-scale models for calculating various fracture response characteristics are proposed in Section 4. Two numerical examples of FGMs, one illustrating random microstructures and the other employing multi-scale and micro-scale fracture analyses, are presented in Section 5. Finally, Section 6 provides conclusions and outlook from this work.

\section{Random microstructure}

\subsection{Level-cut, filtered Poisson field}

Let $(\Omega, \mathcal{F}, P)$ be a probability space, where $\Omega$ is the sample space, $\mathcal{F}$ is the $\sigma$-algebra of subsets of $\Omega$ and $P$ is the probability measure. Defined on the probability triple and indexed by a spatial coordinate $x \in \mathcal{D} \subset \mathbb{R}^{d}$, where $d \geq 1$ is an integer and $\mathcal{D}$ is a bounded subset of $\mathbb{R}^{d}$, consider a real-valued inhomogeneous random field (Grigoriu 2003, Rahman 2008)

$$
Z(\boldsymbol{x}):=\sum_{i=1}^{\mathcal{N}\left(D^{\prime}\right)} Z_{i} h\left(\boldsymbol{\Phi}_{i}\left(\boldsymbol{x}-\boldsymbol{\Gamma}_{i}\right)\right)
$$

where $\mathcal{N}\left(\mathcal{D}^{\prime}\right)$ is an inhomogeneous Poisson field with spatially varying intensity function $\lambda(\boldsymbol{x})$ on $\mathcal{D}^{\prime}$ such that Poisson points $\left\{\boldsymbol{\Gamma}_{i}\right\}$ falling on the set difference $\mathbb{R}^{d} \backslash \mathcal{D}^{\prime}$ do not contribute to the value of $Z, h$ is a non-negative kernel function, $\left\{Z_{i}\right\}$ is a collection of independently distributed real-valued random variables and $\left\{\boldsymbol{\Phi}_{i}\right\}$ is a collection of independently distributed rotation matrices in $\mathbb{R}^{d}$. The filtered Poisson field $Z(\boldsymbol{x})$ can be viewed as the response of a filter with a transfer function at point $\boldsymbol{x}$ that is subjected to a collection of random pulses arriving at Poisson points $\left\{\boldsymbol{\Gamma}_{i}\right\}$.

For an increasing function $g: \mathbb{R} \rightarrow \mathbb{R}$, consider a realvalued translation field (Grigoriu 2003)

$$
Y(\boldsymbol{x}):=g(Z(\boldsymbol{x})),
$$

which describes a memoryless, measurable, non-linear transformation of $Z(x)$. The translation field $Y(x)$ is completely determined by $g$ and $Z(\boldsymbol{x})$, and can follow any marginal distribution, although its correlation structure depends on its marginal distribution and correlation properties of $Z(x)$. Suppose that the mapping in Equation (2) has the form (Grigoriu 2003)

$$
g(z)=I(z ; a):=\left\{\begin{array}{l}
1, z>a \\
0, z \leq a
\end{array},\right.
$$

where $a$ is a deterministic constant, known as level, and $I(z ; a)$ denotes an indicator function. The translation field $Y$ in Equation (2) with $g$ in Equation (3) is referred to as a level-cut random field. The random field $Y(\boldsymbol{x}):=$ $I(Z(x) ; a)$ provides a convenient model for two-phase composites, for example, the subsets $\{\boldsymbol{x} \in \mathcal{D}: Y(\boldsymbol{x})=1\}$ and $\{\boldsymbol{x} \in \mathcal{D}: Y(\boldsymbol{x})=0\}$ define particles (phase 1) and matrix (phase 2), respectively, that can be derived from a $d$-1dimensional contour of $Z(x)$ at level $a$. Figure 1 depicts a schematic illustration of the two subsets when $d=2$ (Figure 1b), obtained from a cut of a generic random field $Z(\boldsymbol{x})$ (Figure 1a). The dark (charcoal) phase indicates particles embedded in the light (light blue) phase representing the matrix. The model results from a direct extension of the level-cut, homogeneous, filtered poisson field developed by Grigoriu (2003). 

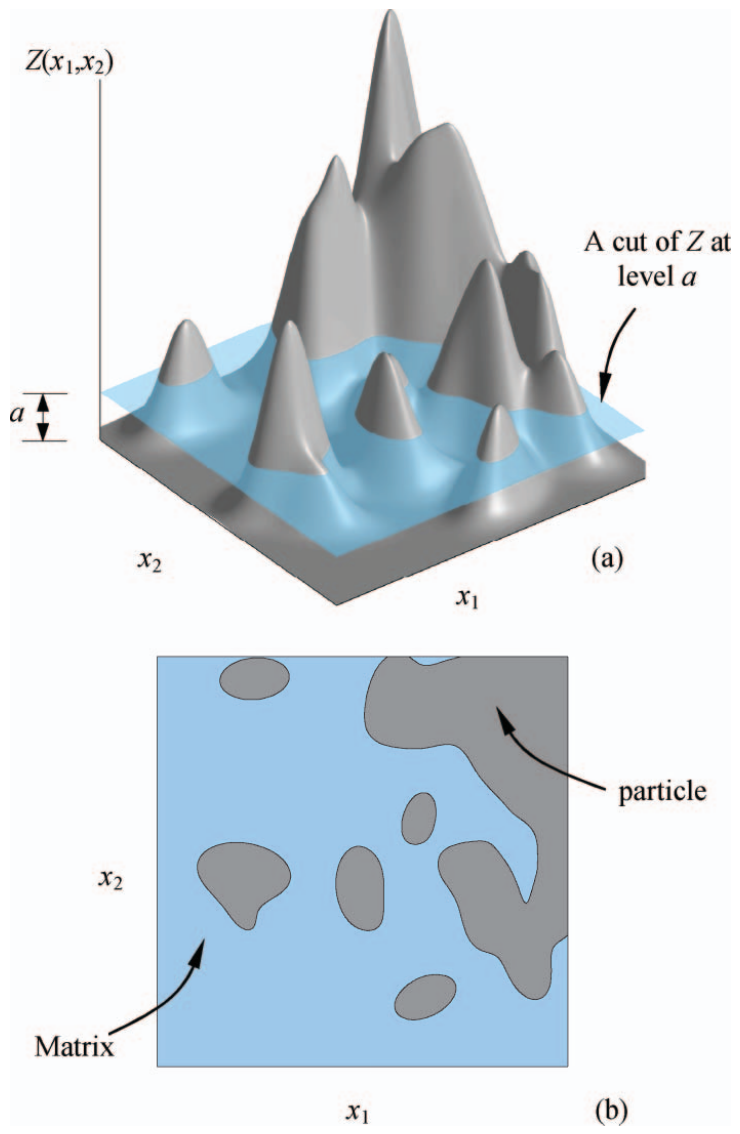

Figure 1. Schematic illustration of a level-cut random field: (a) sample of $Z$ with a cut at level $a$; (b) two subsets of $Y$ obtained from the contour of the sample of $Z$ at level $a$.

\subsection{Correlation functions}

The second-moment characteristics of the level-cut field $Y(\boldsymbol{x})$ in Equations (1-3) are

$$
\mathbb{E}[Y(\boldsymbol{x})]=P[Z(\boldsymbol{x})>a]=p_{1}(\boldsymbol{x})
$$

and

$$
\begin{aligned}
\mathbb{E}\left[Y_{1}(\boldsymbol{x}) Y_{2}(\boldsymbol{x})\right] & =P\left[Z\left(\boldsymbol{x}_{1}\right)>a, Z\left(\boldsymbol{x}_{2}\right)>a\right] \\
& =p_{11}\left(\boldsymbol{x}_{1}, \boldsymbol{x}_{2}\right),
\end{aligned}
$$

where $\mathbb{E}$ is the expectation operator, $p_{1}(\boldsymbol{x})$ is the spatially variant volume fraction of phase 1 and $p_{11}\left(\boldsymbol{x}_{1}, \boldsymbol{x}_{2}\right)$ denotes the translationally variant two-point correlation function. Higher-order moments of $Y(\boldsymbol{x})$, defined by

$$
\begin{aligned}
\mathbb{E}\left[Y_{1}(\boldsymbol{x}) \cdots Y_{k}(\boldsymbol{x})\right] & =P\left[Z\left(\boldsymbol{x}_{1}\right)>a, \ldots, Z\left(\boldsymbol{x}_{k}\right)>a\right] \\
& =p_{1 \cdots 1}\left(\boldsymbol{x}_{1}, \ldots, \boldsymbol{x}_{k}\right),
\end{aligned}
$$

can also be related to the $k$-point correlation function $p_{1 \ldots 1}\left(\boldsymbol{x}_{1}, \ldots, \boldsymbol{x}_{k}\right)$, where $k \geq 1$. Since $Y(\boldsymbol{x})$ is an inhomogeneous field, the correlation function $p_{1 \ldots 1}\left(x_{1}, \ldots, x_{k}\right)$ is also spatially variant, i.e. it explicitly depends on the arguments $\boldsymbol{x}_{1}, \ldots, \boldsymbol{x}_{k}$. It is not possible to analytically derive the second- or higher-order moments of $Y(\boldsymbol{x})$ in a general setting, i.e. when $h,\left\{Z_{i}\right\}$ and $\left\{\boldsymbol{\Phi}_{i}\right\}$ are arbitrarily prescribed. However, these moments are directly related to finite-dimensional distribution functions of $Z(x)$. For instance, when the characteristic function of $\left\{Z\left(\boldsymbol{x}_{1}\right), \ldots, Z\left(\boldsymbol{x}_{n}\right)\right\} ; n \geq 1$ undergoes Fourier transformation, the probability density function of $\left\{Z\left(x_{1}\right), \ldots, Z\left(x_{n}\right)\right\}$ is obtained. Subsequently, the moments of the random fields $Z(\boldsymbol{x})$ and $Y(\boldsymbol{x}):=I(Z(\boldsymbol{x}) ; a)$ can be derived from the density function. See Grigoriu (2003) for further details.

\subsection{Model calibration}

Consider a two-phase particle-matrix microstructure, which can be modelled by $Y$ in Equation (2), where $Z$ is defined in Equation (1). Suppose, estimates of some features of $Y$ are available - for instance, the functions $p_{1}(\boldsymbol{x})$ and $p_{11}\left(\boldsymbol{x}_{1}, \boldsymbol{x}_{2}\right)$ and information on the number of particles and particle geometry. The objective of calibration is to determine parameters of the proposed model such that the second moments of $Y$ in Equations (4) and (5), which are also the first- and second-order probability distributions of $Z$, match known target statistics $\bar{p}_{1}(\boldsymbol{x})$ and $\bar{p}_{11}\left(\boldsymbol{x}_{1}, \boldsymbol{x}_{2}\right)$. If estimates of higher-order moments, such as $k$-point correlation functions $\bar{p}_{1 \cdots 1}\left(\boldsymbol{x}_{1}, \ldots, \boldsymbol{x}_{k}\right) ; k \geq 3$, are available, they can also be incorporated into the calibration process.

Let $\boldsymbol{\theta}$ define a vector of parameters comprising $\lambda(\boldsymbol{x}), h$, $a, Z_{i}$ and $\boldsymbol{\Phi}_{i}$, which are embedded in the proposed random field model. Using any admissible values of these parameters, Monte Carlo simulation can be employed to generate samples of the random field $Y$ and hence calculate its desired moments. Let $p_{1 \cdots 1}(\cdot ; \boldsymbol{\theta})$ represent the $k$-point correlation function of $Y$ corresponding to a selected value of $\boldsymbol{\theta}$. Denote by $\boldsymbol{\theta}^{*}$ the value of $\boldsymbol{\theta}$ that minimises the difference between $p_{1 \ldots 1}(\cdot ; \boldsymbol{\theta})$ and the target $\bar{p}_{1 \cdots 1}\left(\boldsymbol{x}_{1}, \ldots, \boldsymbol{x}_{k}\right)$ for all $k=1, \ldots, K$ in the least squares or other sense, where $K$ is a user-selected positive integer. The optimisation process must consider only feasible values of $\boldsymbol{\theta}$ for which $p_{1 \ldots 1}(\cdot ; \boldsymbol{\theta})$ constitutes a legitimate correlation function. The subspace of feasible values of $\boldsymbol{\theta}$ also depends on the indicator function defined in Equation (2). Most existing models entail calibration processes that are based on matching only the first moment $(K=1)$, i.e. $\bar{p}_{1}(\boldsymbol{x})$, and sometimes also on matching the second moment $(K=2)$, i.e. $\bar{p}_{11}\left(\boldsymbol{x}_{1}, \boldsymbol{x}_{2}\right)$. The proposed model permits a calibration process that can match up to $K$-point correlation functions for any $k \geq 1$ with their corresponding estimates, if available.

Microstructural reconstruction of random heterogeneous materials using specified yet limited correlation functions constitutes an intriguing inverse problem. Since $Y(\boldsymbol{x})$ is non-Gaussian, the statistics $p_{1 \ldots 1}\left(\boldsymbol{x}_{1}, \ldots, \boldsymbol{x}_{k}\right) ; k=$ $1, \ldots, K$, i.e. its 1 - to $K$-point correlation functions, only partially describe its probability law. Consequently, there 
may exist a collection of random fields, referred to as the class of competing models, that have the same aforementioned statistics. The number of competing models can be reduced if $K$ is theoretically made a large number; however, from a practical viewpoint, the value of $K$ is usually decided based on the available information. Nonetheless, a finite value of $K$, no matter how large, will yield a class of competing models with two or more members. If these members have different sample properties, they will generate microstructures with distinct morphological features, pointing to a lack of uniqueness in any reconstruction algorithm.

\subsection{Algorithm}

Once the parameters of the level-cut field $Y(x)$ have been optimised from the calibration process described in the previous subsection, samples of synthetic microstructures can be generated based on the following algorithm:

- Step 1: Define subsets $\mathcal{D}$ and $\mathcal{D}^{\prime}$ of $\mathbb{R}^{d}$. Specify the kernel $h$ and define its parameters.

- Step 2: Generate a sample $n^{*}$ of the homogeneous Poisson random variable $\mathcal{N}\left(\mathcal{D}^{\prime}\right)$, which has a constant intensity $\lambda^{*}=\max _{x \in \mathbb{R}^{d}} \lambda(\boldsymbol{x})$. Generate $n^{*}$ independent uniformly distributed points in $\mathcal{D}^{\prime}$. Denote these points by $\boldsymbol{x}_{i}, i=1, \ldots, n^{*}$.

- Step 3: Perform thinning of the point set obtained in Step 2. In so doing, each point $\boldsymbol{x}_{i}$, independently of the other, is kept with probability $\lambda(\boldsymbol{x}) / \lambda^{*}$, which is equivalent to discarding the point with probability $1-$ $\lambda(\boldsymbol{x}) / \lambda^{*}$. The resulting point pattern of size $n^{* *} \leq n^{*}$ follows the inhomogeneous Poisson field $\mathcal{N}\left(\mathcal{D}^{\prime}\right)$ with intensity function $\lambda(\boldsymbol{x})$. Denote these points by $\boldsymbol{\Gamma}_{i}, i=$ $1, \cdots, n^{* *}$.

- Step 4: Generate $n^{* *}$ independent samples of random rotation matrices $\left\{\boldsymbol{\Phi}_{i}\right\}$ and random variables $\left\{Z_{i}\right\}$. Calculate the corresponding samples of the random fields $Z(\boldsymbol{x})$ from Equation (1) and $Y(\boldsymbol{x}):=I(Z(\boldsymbol{x}) ; a)$ for a specified level $a$. The sample of $Y(x)$ yields a statistically inhomogeneous microstructure.

Further details are available elsewhere (Grigoriu 2003, Rahman 2008).

\section{Stochastic fracture of FGM}

Consider a two-phase, functionally graded, heterogeneous solid with a rectilinear crack and domain $\mathcal{D} \subset \mathbb{R}^{2}$ and a schematic illustration of its microstructure, as shown in Figure 2. The microstructure includes two distinct material phases, phase $p$ (green or dark) and phase $m$ (white or light), denoting particle and matrix constituents, respectively. Both constituents represent isotropic and linear-elastic materials, and the elasticity tensors of the particle and matrix, respec-

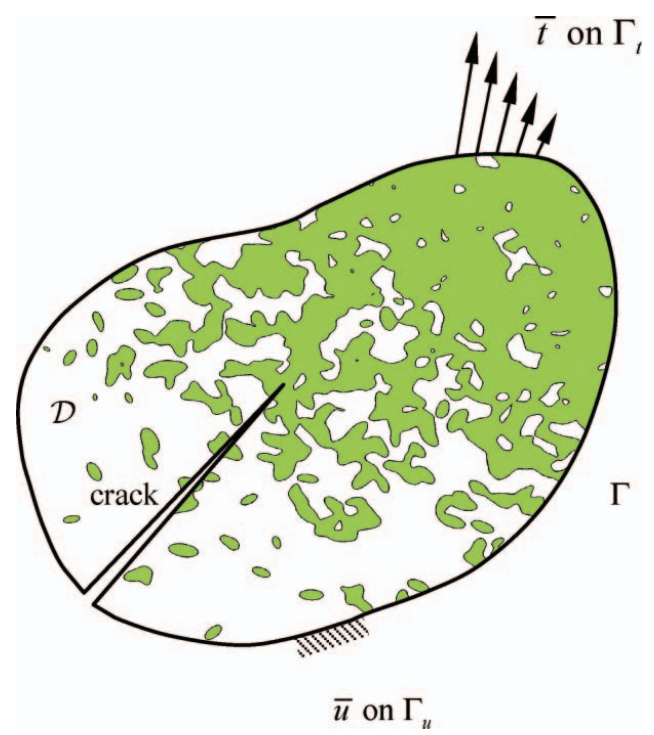

Figure 2. A crack in a functionally graded composite.

tively denoted by $\boldsymbol{C}^{(p)}$ and $\boldsymbol{C}^{(m)}$, are

$$
\boldsymbol{C}^{(i)}=\frac{v_{i} E_{i} \mathbf{1} \otimes \mathbf{1}}{\left(1+v_{i}\right)\left(1-2 v_{i}\right)}+\frac{E_{i}}{1+v_{i}} \boldsymbol{I} ; i=p, m,
$$

where the symbol $\otimes$ denotes tensor product; $E_{i}$ and $v_{i}$ are respectively elastic modulus and Poisson's ratio of phase $i$; and $\mathbf{1}$ and $\boldsymbol{I}$ are respectively second- and fourth-rank identity tensors. The superscripts or subscripts $i=p$ and $i=m$ refer to particle and matrix, respectively. At a spatial point $\boldsymbol{x} \in \mathcal{D}$ in the macroscopic length scale, let $\phi_{p}(\boldsymbol{x})$ and $\phi_{m}(\boldsymbol{x})$ respectively denote the volume fractions of particle and matrix. Each volume fraction is bounded between 0 and 1 and satisfies the constraint: $\phi_{p}(\boldsymbol{x})+\phi_{m}(\boldsymbol{x})=1$. The crack faces are traction-free, and there is perfect bonding between matrix and particles.

For a quasi-static problem with small displacements and strains, the variational or weak form of equilibrium equations and boundary conditions is

$$
\begin{gathered}
\int_{\mathcal{D}}(\boldsymbol{C}(\boldsymbol{x}): \boldsymbol{\varepsilon}): \delta \boldsymbol{\varepsilon} d \mathcal{D}-\int_{\mathcal{D}} \boldsymbol{b} \cdot \delta \boldsymbol{u} d \mathcal{D}-\int_{\Gamma_{t}} \overline{\boldsymbol{t}} \cdot \delta \boldsymbol{u} d \Gamma \\
-\sum_{x_{K} \in \Gamma_{u}} \boldsymbol{f}\left(\boldsymbol{x}_{K}\right) \cdot \delta \boldsymbol{u}\left(\boldsymbol{x}_{K}\right) \\
-\sum_{x_{K} \in \Gamma_{u}} \delta \boldsymbol{f}\left(\boldsymbol{x}_{K}\right) \cdot\left[\boldsymbol{u}\left(\boldsymbol{x}_{K}\right)-\overline{\boldsymbol{u}}\left(\boldsymbol{x}_{K}\right)\right]=0
\end{gathered}
$$

where $\boldsymbol{u}: \mathcal{D} \rightarrow \mathbb{R}^{2}$ is the displacement vector; $\boldsymbol{\sigma}=\boldsymbol{C}(\boldsymbol{x})$ : $\boldsymbol{\varepsilon}$ is the Cauchy stress tensor with $\boldsymbol{C}(\boldsymbol{x})$ and $\boldsymbol{\varepsilon}$ denoting the spatially variant elasticity tensor and strain tensor, respectively; $\Gamma_{t}$ and $\Gamma_{u}$ are two disjoint portions of the boundary, where the traction vector $\overline{\boldsymbol{t}}$ and displacement $\overline{\boldsymbol{u}}$ are prescribed; $f^{T}\left(\boldsymbol{x}_{K}\right)$ is the vector of reaction forces at the 
constrained node $K$ on $\Gamma_{u}$; and symbols '.' and ':' denote dot product and tensor contraction, respectively. The discretisation of the weak form, Equation (8), depends on how the elasticity tensor $\boldsymbol{C}(\boldsymbol{x})$ is defined, i.e. how the elastic properties of constituent material phases and their gradation characteristics are described. In a following subsection, various multi-scale and uniscale models are presented to approximate $\boldsymbol{C}(\boldsymbol{x})$. Nonetheless, a numerical method, e.g. the finite-element method (FEM), is generally required to solve the discretised weak form, providing various response fields of interest.

\subsection{Statistical models of random input}

Uncertainties in FGM fracture can come from a variety of sources - for instance, the FGM microstructure, constituent material properties, boundary conditions, crack geometry and structural geometry. Microstructural uncertainty includes randomness in particle volume fractions, spatial arrangements of particles, and size, shape and orientation properties of particles. In this work, however, the sources of uncertainties are limited to particle volume fraction, particle location and constituent material properties. Further details of uncertainty modelling in FGM are given by Chakraborty and Rahman (2008).

The total number of random variables depends on the multi-scale or micro-scale analysis described in the following section. In general, the random variables include: (1) $M$ random variables $\left\{Z_{p, 1}, \ldots, Z_{p, M}\right\}$ due to the discretisation of the random field $\phi_{p}(\boldsymbol{x})$; (2) a Poisson random variable $\mathcal{N}$ and resulting $2 \mathcal{N}$ random coordinates $\left\{U_{i, 1}, U_{i, 2}\right\} ; i=$ $1, \ldots, \mathcal{N}$ of the centroids of particles in $\mathcal{D}$; and (3) four random constituent properties $\left\{E_{p}, E_{m}, v_{p}, v_{m}\right\}$. Depending on the multi-scale or micro-scale model, some or all of these random variables are required for stochastic fracture analysis. Nevertheless, the maximum number of random variables is $N=M+2 \mathcal{N}+5$. In other words, a vector $\boldsymbol{R} \in \mathbb{R}^{N}$, comprising random variables from all possible sources, characterises input uncertainty in an FGM and is completely described by its joint probability density function (PDF) $f_{\boldsymbol{R}}(\boldsymbol{r})$, where $\boldsymbol{r}$ is a realisation of $\boldsymbol{R}$.

\subsection{Crack-driving forces and reliability}

A major objective of stochastic fracture-mechanics analysis is to find probabilistic characteristics of stress-intensity factors (SIFs) $K_{I}(\boldsymbol{R})$ and $K_{I I}(\boldsymbol{R})$ for modes I and II, respectively, due to uncertain input $\boldsymbol{R}$. For a given input, the standard FEM can be employed to solve the discretised weak form (Equation (8)), leading to the calculation of SIFs and other crack-driving forces. Let $y(\boldsymbol{R})$ describe a generic crack-driving force or a relevant performance function involving crack-driving forces for a given fracture problem of interest. In general, the multivariate function $y: \mathbb{R}^{N} \rightarrow \mathbb{R}$ is implicit, is not analytically available and can only be viewed as a high-dimensional input-output mapping, where the evaluation of the output function $y$ for a given input $\boldsymbol{r}$ requires expensive finite-element analysis. Therefore, methods employed in stochastic analysis must be capable of generating accurate probabilistic characteristics of $y(\boldsymbol{R})$ with an acceptably small number of output function evaluations.

Suppose that a failure is defined when the crack propagation is initiated at a crack tip, i.e. when $K_{\text {eff }}(\boldsymbol{R}):=$ $h_{m}\left(K_{I}(\boldsymbol{R}), K_{I I}(\boldsymbol{R})\right)>K_{I c}$, where $K_{\text {eff }}(\boldsymbol{R})$ is an effective SIF with $h_{m}$ depending on a selected mixed-mode theory, and $K_{I c}$ is a relevant mode-I fracture toughness of the material measured in terms of SIF. This requirement cannot be satisfied with certainty, since $K_{I}(\boldsymbol{R})$ and $K_{I I}(\boldsymbol{R})$ are both dependent on $\boldsymbol{R}$ which is random, and $K_{I c}$ itself may be a random variable or field. Hence, the performance of a cracked FGM should be evaluated by the reliability or its complement, the conditional probability of failure $P_{F}$, defined as the multi-fold integral

$$
P_{F}\left(K_{I c}\right):=P[y(\boldsymbol{R})<0]:=\int_{R^{N}} I_{y}(\boldsymbol{r}) f_{\boldsymbol{R}}(\boldsymbol{r}) d \boldsymbol{r}
$$

where

$$
y(\boldsymbol{R})=K_{I c}-h_{m}\left(K_{I}(\boldsymbol{R}), K_{I I}(\boldsymbol{R})\right)
$$

is a multivariate performance function that depends on the random input $\boldsymbol{R}$ and $I_{y}(\boldsymbol{r})$ is another indicator function, taking values of one when $y(\boldsymbol{r})<0$ and zero when $y(\boldsymbol{r})>0$. In this work, the maximum circumferential stress theory was invoked to describe mixed-mode fracture initiation (Anderson 2005).

The evaluation of the multi-dimensional integral in Equation (9), either analytically or numerically, is not possible because the total number of random variables $N$ is large, $f_{\boldsymbol{R}}(\boldsymbol{r})$ is generally non-Gaussian, and $y(\boldsymbol{r})$ is a highly non-linear function of $\boldsymbol{r}$. In this work, the recently developed dimensional decomposition method (Xu and Rahman 2005) and direct Monte Carlo simulation were employed for calculating the probabilistic characteristics of crack-driving forces and the probability of fracture initiation.

\section{Multi-scale and micro-scale analyses}

The FGM microstructure schematically illustrated in Figure 2 contains discontinuities in material properties at the interfaces between the matrix and particles. However, it is unclear how such discontinuities will affect the calculation of a SIF. In addition, a mathematically sharp crack tip, which has no geometric dimensions, is located in either the matrix or the particle phase. By employing an effective elastic modulus, commonly used for deriving the energy release rate of a cracked solid, may yield inadequate estimates of the resultant SIFs, particularly if there exists a significant mismatch between the matrix and particle properties. However, 
far away from the crack tip, where the effect of the crack-tip singularity dies off rapidly, individual constituent material properties may not be needed, and an appropriately derived effective material property should suffice. From the above discussion, there exist two major approaches with respect to defining the material property for fracture analysis of an FGM cracked structure (Chakraborty and Rahman 2008). One approach involves multi-scale analysis, which requires performing stress analysis in both microscopic and macroscopic length scales. The other approach entails a uniscale analysis that is solely based on the microscopic length scale. The words 'micro-scale' and 'macro-scale' analyses refer to stress analyses entailing presence and absence, respectively, of explicit particle geometry in the domain of the solid. In this work, three multi-scale and a micro-scale models, schematically illustrated in Figure 3, were examined.

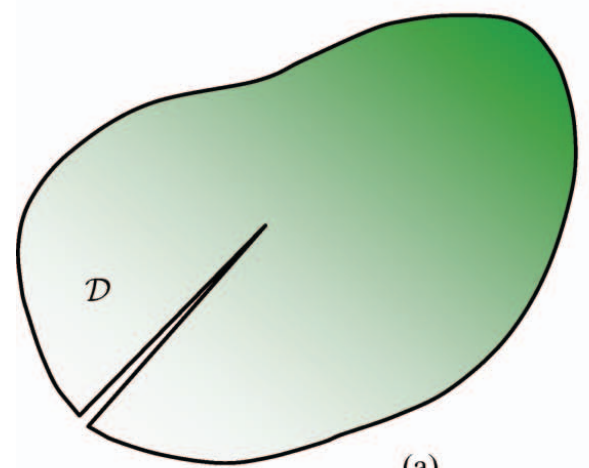

(a)

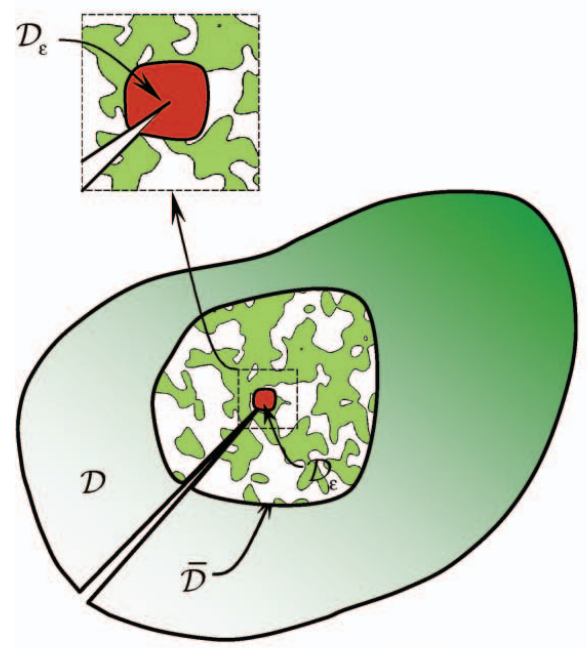

(c)

\subsection{Multi-scale models}

The multi-scale analysis can be conducted in several ways depending on how the information derived from the lower scale (micro-scale) transfers to or interacts with the analysis in the higher scale (macro-scale). However, the mechanics and stochastics involved vary depending on the multi-scale model selected, and are described as follows.

All multi-scale models presented here require continuously varying effective properties, defined either completely or partially in the domain of the solid. Typically, a micromechanical analysis is performed to predict response fields of interest in the micro-scale, followed by a homogenisation to produce the continuously varying effective properties. Let $\bar{C}(\boldsymbol{x})$ denote the continuously varying effective elasticity tensor of the FGM at a point $x \in \mathcal{D}$,

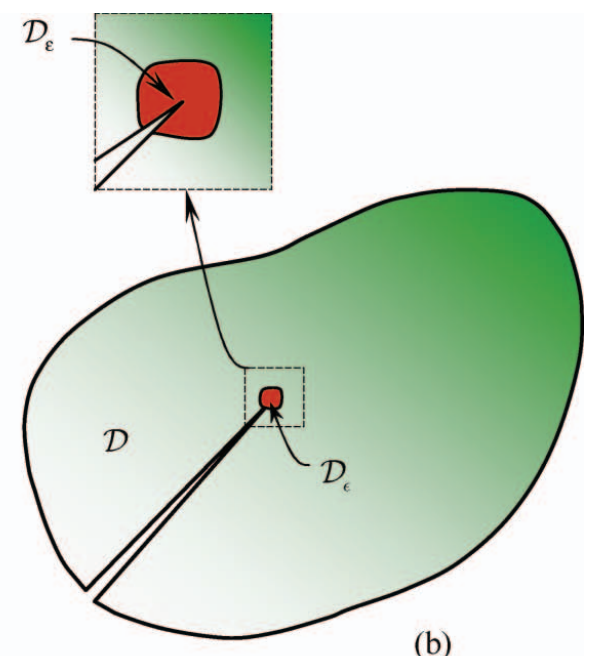

(b)

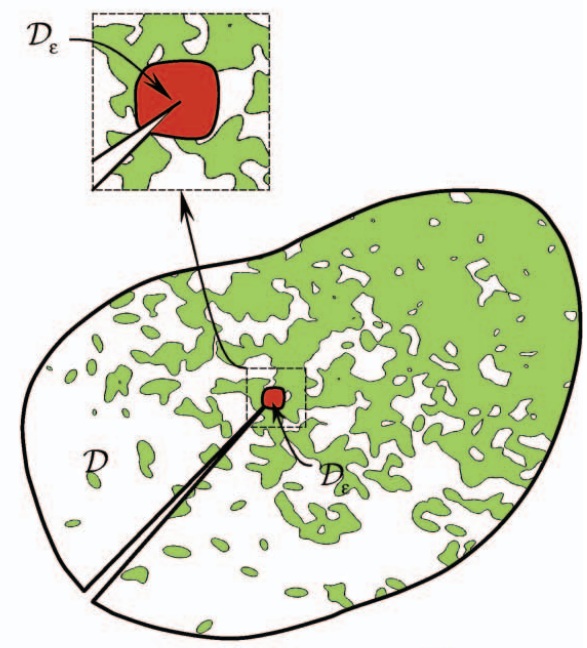

(d)

Figure 3. Schematics of various models: (a) sequential; (b) invasive; (c) concurrent; (d) micro-scale. 
expressed by

$$
\bar{C}(\boldsymbol{x})=\frac{\bar{v}(\boldsymbol{x}) \bar{E}(\boldsymbol{x}) \mathbf{1} \otimes \mathbf{1}}{[1+\bar{v}(\boldsymbol{x})][1-2 \bar{v}(\boldsymbol{x})]}+\frac{\bar{E}(\boldsymbol{x})}{1+\bar{v}(\boldsymbol{x})} \boldsymbol{I},
$$

where the effective elastic modulus $\bar{E}(\boldsymbol{x})$ and effective Poisson's ratio $\bar{v}(\boldsymbol{x})$ both depend on $\boldsymbol{x} \in \mathcal{D}$. Using classical micromechanics - for instance, the self-consistent model, the Mori-Tanaka model, the mean-field theory and others (Mura 1991) - the effective elasticity tensor $\bar{C}(\boldsymbol{x})$ can be readily calculated.

\subsubsection{Sequential multi-scale model}

The sequential or hierarchical multi-scale model adopted in the present work includes a three-step serial process (Figure 3a). First, the effective tensor $\bar{C}(\boldsymbol{x})$ is calculated using micromechanics based homogenisation. Second, a macromechanical stress analysis based on the effective elasticity tensor is conducted to generate the field solutions. In this step, the weak form, described by Equation (8), is discretised using

$$
C(x) \cong \bar{C}(x), x \in \mathcal{D}
$$

and then solved by the FEM, leading to stress, strain and displacement fields in the macroscopic length scale.

Third, a macromechanical fracture-mechanics analysis using an interaction integral $\tilde{M}^{(1,2)}$ for inhomogeneous materials yields the mixed-mode SIFs (Rao and Rahman 2003). The SIFs can be obtained from (Rao and Rahman 2003)

$$
K_{i} \cong \frac{1}{2} \tilde{M}^{(1, i)} \bar{E}_{\mathrm{tip}}^{*} ; i=I, I I,
$$

where $\bar{E}_{\text {tip }}^{*}$, equal to $\bar{E}\left(\boldsymbol{x}_{\text {tip }}\right)$ for the plane stress and $\bar{E}\left(\boldsymbol{x}_{\text {tip }}\right) /\left[1-\bar{v}_{\text {tip }}^{2}\left(\boldsymbol{x}_{\text {tip }}\right)\right]$ for the plane strain conditions, is the effective generalised modulus of the FGM at the crack tip $\boldsymbol{x}_{\text {tip }}$, and $\tilde{M}^{(1, I)}$ and $\tilde{M}^{(1, I)}$ are two interaction integrals for modes I and II, respectively.

\subsubsection{Invasive multi-scale model}

The invasive multi-scale model also involves a three-step serial process: (1) a micromechanical analysis to predict response fields of interest in the micro-scale, followed by a homogenisation to produce smoothly varying effective properties in the macro-scale; (2) a micro-macromechanical stress analysis based on locally homogeneous material properties to generate the field solutions; and (3) a macromechanical fracture analysis based on locally homogeneous material properties to calculate the mixed-mode SIFs.

In the invasive multi-scale model, the effective elasticity tensor is also calculated from a micromechanical ho- mogenisation, as in the sequential model. However, the weak form, Equation (8), is discretised and then solved using

$$
\boldsymbol{C}(\boldsymbol{x}) \cong \begin{cases}\boldsymbol{C}^{(p)}, & \text { if } \boldsymbol{x} \in \mathcal{D}_{\varepsilon} \text { and } \boldsymbol{x}_{\text {tip }} \in \mathcal{D}_{p} \\ \boldsymbol{C}^{(m)}, & \text { if } \boldsymbol{x} \in \mathcal{D}_{\varepsilon} \text { and } \boldsymbol{x}_{\text {tip }} \in \mathcal{D}_{m}, \\ \overline{\boldsymbol{C}}(\boldsymbol{x}), & \text { if } \boldsymbol{x} \in \mathcal{D} \backslash \mathcal{D}_{\varepsilon}\end{cases}
$$

where $\mathcal{D}_{\varepsilon} \subset \mathcal{D}$ is a small bounded sub-domain surrounding the crack tip, and $\mathcal{D}_{p} \subset \mathcal{D}$ and $\mathcal{D}_{m} \subset \mathcal{D}$ are particle and matrix sub-domains, respectively. According to Equation (14), discrete material properties of either the particle $\left(\boldsymbol{C}^{(p)}\right)$ or the matrix $\left(\boldsymbol{C}^{(m)}\right)$ are assigned to a small subdomain surrounding the crack tip and continuously varying effective material properties $(\overline{\boldsymbol{C}}(\boldsymbol{x}))$ are defined on elsewhere, as shown in Figure $3 b$. Therefore, discontinuities in material properties exist at the boundary of $\mathcal{D}_{\varepsilon}$.

Since the material representation is locally homogeneous, but constant for $x \in \mathcal{D}_{\varepsilon}$, the mixed-mode SIFs can be calculated from the interaction integral applicable to homogeneous materials. If the crack-tip contour is restricted to lie inside $\mathcal{D}_{\varepsilon}$, the interaction integral for a locally homogeneous material (Rao and Rahman 2003), $M^{(1,2)}$, can be easily calculated. The integral $M^{(1,2)}$ is different when $\boldsymbol{C}(\boldsymbol{x})=\boldsymbol{C}^{(p)} ; \boldsymbol{x} \in \mathcal{D}_{\varepsilon}$, e.g. $\boldsymbol{M}_{p}^{(1,2)}$ and when $\boldsymbol{C}(\boldsymbol{x})=\boldsymbol{C}^{(m)} ; \boldsymbol{x} \in \mathcal{D}_{\varepsilon}$, e.g. $\boldsymbol{M}_{m}^{(1,2)}$. Then, the SIFs can be calculated from

$$
K_{i} \cong\left\{\begin{array}{ll}
\frac{1}{2} M_{p}^{(1, i)} E_{p}^{*}, & \text { if } \boldsymbol{x}_{\text {tip }} \in \mathcal{D}_{p} \\
\frac{1}{2} M_{m}^{(1, i)} E_{m}^{*}, & \text { if } \boldsymbol{x}_{\text {tip }} \in \mathcal{D}_{m}
\end{array} ; \quad i=I, I I .\right.
$$

Compared with the sequential (Equation (13)) model, the invasive model (Equation (15)) utilises more accurate crack-tip conditions and hence should provide improved estimates of SIFs over the former model. However, it is unclear whether the improvements in the invasive model are adequate for calculating the failure probability.

\subsubsection{Concurrent multi-scale model}

The concurrent model includes both continuous and discrete material representations and requires a combined micromechanical and macromechanical stress analysis. Depicted in Figure 3c, consider an arbitrary bounded subdomain $\overline{\mathcal{D}} \subseteq \mathcal{D}$, which contains a finite number of particles embedded in the matrix. The number of particles falling in $\overline{\mathcal{D}}^{\prime}$ is $\overline{\mathcal{N}}=\mathcal{N}\left(\overline{\mathcal{D}}^{\prime}\right)$, where $\overline{\mathcal{D}}^{\prime} \subset \mathbb{R}^{2}$ is a bounded subset such that points of $\overline{\mathcal{N}}$ falling in $\mathbb{R}^{2} \backslash \overline{\mathcal{D}}^{\prime}$ do not contribute to particles in $\overline{\mathcal{D}}$. The integer-valued random variable $\overline{\mathcal{N}}$ also follows a Poisson distribution with the same intensity function $\lambda(\boldsymbol{x})$. The sub-domain $\overline{\mathcal{D}}$, once defined, is statistically filled with particles in the matrix, and the remaining 
sub-domain $\mathcal{D} \backslash \overline{\mathcal{D}}$ is assigned a continuously varying effective elasticity tensor $\overline{\boldsymbol{C}}(\boldsymbol{x})$, derived from a suitable micromechanical homogenisation as in previous multiscale models. According to the concurrent model, Equation (8) is discretised and solved using

$$
\boldsymbol{C}(\boldsymbol{x}) \cong \begin{cases}\boldsymbol{C}^{(p)}, & \text { if } \boldsymbol{x} \in \overline{\mathcal{D}} \text { and } \boldsymbol{x} \in \mathcal{D}_{p} \\ \boldsymbol{C}^{(m)}, & \text { if } \boldsymbol{x} \in \overline{\mathcal{D}} \text { and } \boldsymbol{x} \in \mathcal{D}_{m} \\ \overline{\boldsymbol{C}}(\boldsymbol{x}), & \text { if } \boldsymbol{x} \in \mathcal{D} \backslash \overline{\mathcal{D}}\end{cases}
$$

Therefore, discontinuities in material properties exist at the interfaces between $\overline{\mathcal{D}}$ and $\mathcal{D} \backslash \overline{\mathcal{D}}$ and between $\mathcal{D}_{p}$ and $\mathcal{D}_{m}$.

Since the material representation in $\overline{\mathcal{D}}$ is discrete, the calculation of the resulting SIFs in the concurrent model is not straightforward. The interaction integral $\tilde{M}^{(1,2)}$ or $M^{(1,2)}$, conveniently exploited in the former multi-scale models, requires either continuously varying or constant material properties inside the domain of a crack-tip contour. However, if the idea of the small sub-domain $\mathcal{D}_{\varepsilon} \subset \overline{\mathcal{D}} \subseteq \mathcal{D}$, introduced in the previous subsection, is borrowed in slightly modifying the elasticity tensor, for instance,

$$
\boldsymbol{C}(\boldsymbol{x}) \cong \begin{cases}\boldsymbol{C}^{(p)}, & \text { if }\left(\boldsymbol{x} \in \mathcal{D}_{\varepsilon} \text { and } \boldsymbol{x}_{\text {tip }} \in \mathcal{D}_{p}\right) \text { or } \\ & \text { if }\left(\boldsymbol{x} \in \overline{\mathcal{D}} \backslash \mathcal{D}_{\varepsilon} \text { and } \boldsymbol{x} \in \mathcal{D}_{p}\right) \\ \boldsymbol{C}^{(m)}, & \text { if }\left(\boldsymbol{x} \in \mathcal{D}_{\varepsilon} \text { and } \boldsymbol{x}_{\text {tip }} \in \mathcal{D}_{m}\right) \text { or } \\ \overline{\boldsymbol{C}}(\boldsymbol{x}), & \text { if }\left(\boldsymbol{x} \in \mathcal{\mathcal { D }} \backslash \mathcal{D}_{\varepsilon} \text { and } \boldsymbol{x} \in \overline{\mathcal{D}},\right.\end{cases}
$$

the material representation in the concurrent model remains locally homogeneous, rendering the interaction integral method applicable. Applying Equation (17) in solving the discretised weak form yields the $M$-integrals, $M_{p}^{(1,2)}$ and $M_{m}^{(1,2)}$, when $\boldsymbol{C}(\boldsymbol{x})=\boldsymbol{C}^{(p)} ; \boldsymbol{x} \in \mathcal{D}_{\varepsilon}$ and $\boldsymbol{C}(\boldsymbol{x})=\boldsymbol{C}^{(m)} ; \boldsymbol{x} \in$ $\mathcal{D}_{\varepsilon}$, respectively. The SIFs are subsequently calculated using Equation (15). Although the same fracture-mechanics equations are used, the SIFs obtained from the concurrent model should be different than those from the invasive model. This is because the $M$-integrals resulting from Equation (17) (concurrent model) are different than those calculated from Equation (14) (invasive model). The discrete particles in $\overline{\mathcal{D}}$, if they produce any effect, should influence the $M$-integrals obtained in the concurrent model. No such effect is accounted for in the invasive model. The magnitude of the effect, however, depends on the relative size of $\overline{\mathcal{D}}$. If the size of $\overline{\mathcal{D}}$ is shrunk to approach the size of $\mathcal{D}_{\varepsilon}$, the concurrent model degenerates to the invasive model. If the size of $\overline{\mathcal{D}}$ is expanded to approach the size of $\mathcal{D}$, the model no longer remains a multi-scale model, but becomes a uniscale model, which is explained in the next subsection.

\subsection{Micro-scale model}

The micro-scale model is a straightforward uniscale model, where $\mathcal{N}$ discrete particles are dispersed in the matrix, as shown in Figure $3 \mathrm{~d}$. The number of particles falling in $\mathcal{D}^{\prime}$ is $\mathcal{N}\left(\mathcal{D}^{\prime}\right)$, where $\mathcal{D}^{\prime} \subset \mathbb{R}^{2}$ is a bounded subset such that the points of $\mathcal{N}$ falling in $\mathbb{R}^{2} \backslash \mathcal{D}^{\prime}$ do not contribute to the particles in $\mathcal{D}$. For the particle-matrix composite system, a micro-scale stress analysis is performed, where by introducing the small sub-domain $\mathcal{D}_{\varepsilon} \subset \mathcal{D}$, as done in the concurrent model, the modified elasticity tensor

$$
\boldsymbol{C}(\boldsymbol{x}) \cong \begin{cases}\boldsymbol{C}^{(p)}, & \text { if }\left(\boldsymbol{x} \in \mathcal{D}_{\varepsilon} \text { and } \boldsymbol{x}_{\text {tip }} \in \mathcal{D}_{p}\right) \text { or } \\ & \text { if }\left(\boldsymbol{x} \in \mathcal{D} \backslash \mathcal{D}_{\varepsilon} \text { and } \boldsymbol{x} \in \mathcal{D}_{p}\right) \\ \boldsymbol{C}^{(m)}, & \text { if }\left(\boldsymbol{x} \in \mathcal{D}_{\varepsilon} \text { and } \boldsymbol{x}_{\text {tip }} \in \mathcal{D}_{m}\right) \text { or } \\ & \text { if }\left(\boldsymbol{x} \in \mathcal{D} \backslash \mathcal{D}_{\varepsilon} \text { and } \boldsymbol{x} \in \mathcal{D}_{m}\right)\end{cases}
$$

also leads to a local homogenisation; therefore, the $M$ integrals can be readily calculated. Subsequently, the SIFs are calculated by invoking Equation (15). The effect of discrete particles in the entire domain $\mathcal{D}$ is propagated to the $M$-integrals obtained in the micro-scale model. Such effect is also accounted for in the concurrent multi-scale model, but only from particles embedded in the sub-domain $\overline{\mathcal{D}}$.

In summary, three multi-scale models and a micro-scale model have been presented to solve the stochastic fracturemechanics problem described in Section 3. The multi-scale models employ effective material properties whenever possible and include further assumptions or approximations to help solve the problem economically. In contrast, the microscale model constitutes a brute-force approach that employs a discrete particle-matrix system to solve the problem as accurately as possible, considered in the present work. Therefore, the micro-scale model is the most expensive model studied, but it is required to evaluate the accuracy and efficiency of the multi-scale models.

\section{Numerical examples}

\subsection{Microstructures of FGM}

Consider a $10 \times 10$, two-dimensional, FGM specimen, where fully penetrable elliptical or circular particles with random lengths and widths are embedded in the matrix. For the inhomogeneous Poisson field, two types of intensity function associated with diagonal and centrifugal variations of the particle locations were defined. The kernel function was chosen such that the particles have elliptical shapes for the diagonal variation or circular shapes for the centrifugal variation. Figure 4a shows three samples of the level-cut, filtered Poisson field obtained from three distinct levels, $a=0.1,1$ and 5, representing synthetic samples of FGM microstructures with a diagonally varying intensity function, where the particles have a target aspect ratio of 

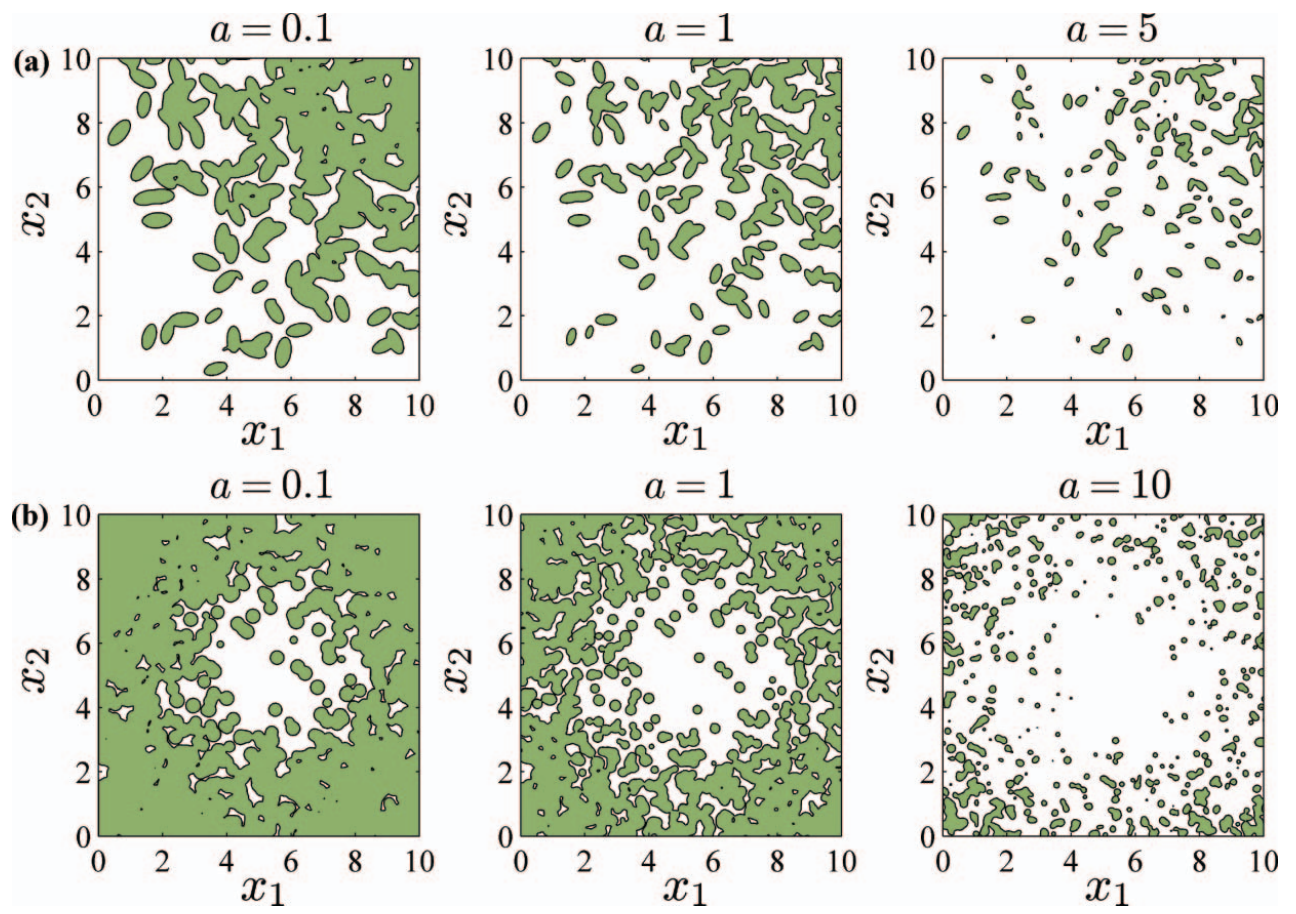

Figure 4. Samples of two-dimensional FGM microstructures: (a) diagonal intensity; (b) centrifugal intensity.

two, but their sizes are random. Figure $4 \mathrm{~b}$ demonstrates the versatility of the proposed random field model by depicting FGM microstructures derived from the centrifugal intensity function. In both variations, the number and sizes of the particles in these composites decrease when the magnitude of the level $a$ increases. Therefore, it is possible to calibrate $a$, leading to a synthetic microstructure with the desired statistical properties.

Consider a $10 \times 10 \times 10$, three-dimensional, FGM specimen, where fully penetrable spherical or disk-shaped particles entailing random sizes and/or orientations are embedded in the matrix. A diagonally varying intensity function characterises the inhomogeneous Poisson field, where two values of the Poisson intensity parameter $c, 0.0005$ and 0.005 , were selected to represent low and high intensities, respectively. Figures $5 \mathrm{a}-5 \mathrm{~d}$ exhibit four samples of two-phase FGMs, obtained for level $a=0.1$. The microstructures in Figures $5 \mathrm{a}$ and $5 \mathrm{~b}$, which include spherical particles, respectively, correspond to low $(c=0.0005)$ and high $(c=0.005)$ values of the Poisson intensity. The higher the value of the Poisson intensity, the higher the particle density, resulting in frequent intersections among particles. The samples in Figures $5 \mathrm{c}$ and $5 \mathrm{~d}$ contain disk-shaped particles, also generated using the same two intensities. The significant difference between particle properties in Figures $5 \mathrm{a}-5 \mathrm{~d}$ demonstrates once again the capability of the proposed model in generating a broad range of two-phase inhomogeneous microstructures.

\subsection{Fracture of FGM}

Consider a $16 \mathrm{~cm} \times 16 \mathrm{~cm}$, two-dimensional, square, FGM specimen, which contains randomly dispersed, fully penetrable, circular, silicon carbide $(\mathrm{SiC})$ particles (radius $=$ $0.48 \mathrm{~cm}$ ) in an aluminium (Al) matrix, as shown in Figure 6 . The specimen contains an $8-\mathrm{cm}$ long edge crack that is subjected to two far-field stresses $\sigma^{\infty}=\tau^{\infty}=1 \mathrm{kN} / \mathrm{cm}^{2}$. The sub-domain $\overline{\mathcal{D}}=16 \kappa \mathrm{cm} \times 16 \kappa \mathrm{cm}$, where $\kappa=0.25$ or 0.5 . The particle volume fraction, which varies only along the horizontal coordinate, is an inhomogeneous, Beta, random field (Chakraborty and Rahman 2007). The material phases $\mathrm{SiC}$ and $\mathrm{Al}$ are both linear-elastic and isotropic. However, their elastic moduli $E_{\mathrm{SiC}}$ and $E_{\mathrm{Al}}$ and the Poisson's ratios $v_{\mathrm{SiC}}$ and $v_{\mathrm{Al}}$ are random variables; their means, coefficient of variations (COV) and probability density functions are listed in Table 1. For the sequential and invasive multi-scale models an efficient stochastic method, known as the dimensional decomposition method (Xu and Rahman 2005), was employed, while the direct Monte Carlo simulation was used for the concurrent multi-scale and micro-scale models. The sample size for both the decomposition and direct Monte Carlo simulation is 10,000 . The finite-element analyses were performed by 


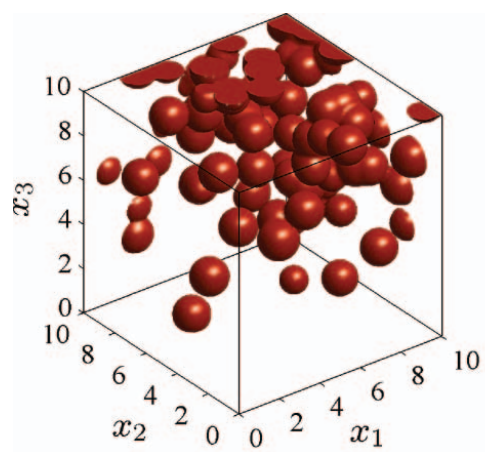

(a)

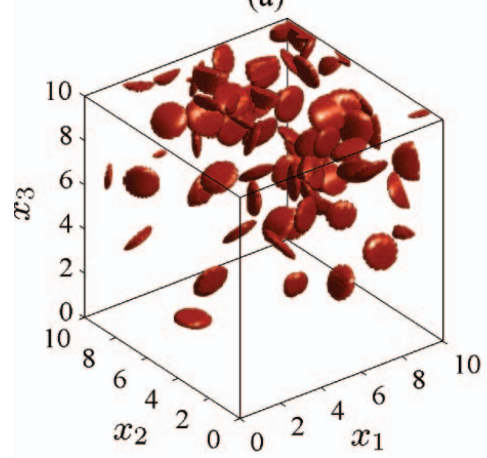

(c)

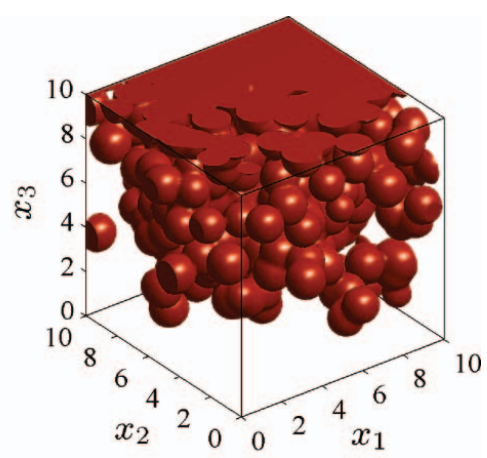

(b)

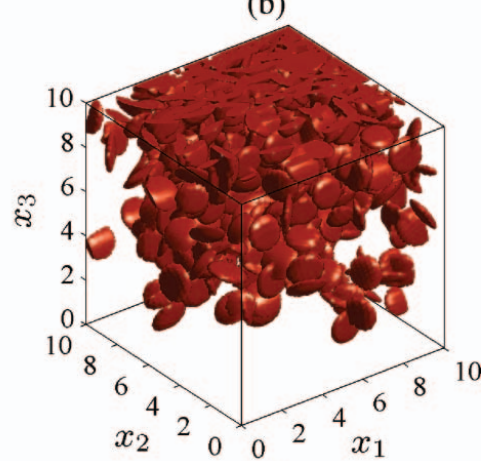

(d)

Figure 5. Samples of three-dimensional FGM microstructures with diagonally varying intensity functions: (a) spherical ( $c=0.0005)$, (b) spherical ( $c=0.005)$; (a) disk-shaped ( $c=0.0005)$, (b) disk-shaped $(c=0.005)$.

the commercial code ABAQUS (2006). The effective properties required by all multi-scale models were calculated using the Mori-Tanaka approximation.

\subsubsection{Results}

Figures $7 \mathrm{a}-7 \mathrm{~d}$ plot von Mises stress contours for the same FGM sample, generated from the sequential, invasive, concurrent and micro-scale models, respectively. The overall

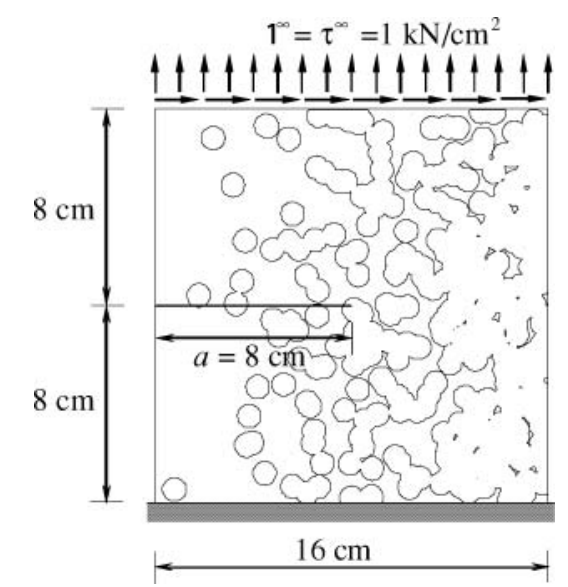

Figure 6. An edge-cracked FGM specimen under mixed-mode loading and deformation (particle radius $=0.48 \mathrm{~cm}$ ). stress responses from all multi-scale and micro-scale models, indicated by the contour patterns, are similar. However, there are also differences in the local stress fields that may have significant implications in determining crack-driving forces and eventually in reliability predictions by various models. The results pertaining to fracture reliability are presented next.

Table 2 lists the second-moment statistics of $K_{I}$ and $K_{I I}$ by various multi-scale and micro-scale models examined in this work. Among various models, the statistics from the micro-scale model, which requires the fewest approximations, were treated as the reference solutions. From Table 2, the sequential and invasive models show reasonably good agreement when comparing the mean values of $K_{I}$ and $K_{I I}$ with those of the reference model. The concurrent model with $\kappa=0.25$ or $\kappa=0.5$ provides better estimates of the

Table 1. Statistical properties of constituents.

\begin{tabular}{lccc}
\hline Elastic property ${ }^{(a)}$ & Mean & COV (\%) & PDF \\
\hline$E_{\mathrm{SiC}}, \mathrm{GPa}$ & 419.2 & 15 & Lognormal \\
$E_{\mathrm{Al}}, \mathrm{GPa}$ & 69.7 & 10 & Lognormal \\
$\nu_{\mathrm{SiC}}$ & 0.19 & 15 & Lognormal \\
$\nu_{\mathrm{Al}}$ & 0.34 & 10 & Lognormal \\
\hline
\end{tabular}

${ }^{(a)} E_{p}=E_{\mathrm{SiC}} ; E_{m}=E_{\mathrm{Al}} ; v_{p}=v_{\mathrm{SiC}} ; v_{m}=v_{\mathrm{Al}}$. 

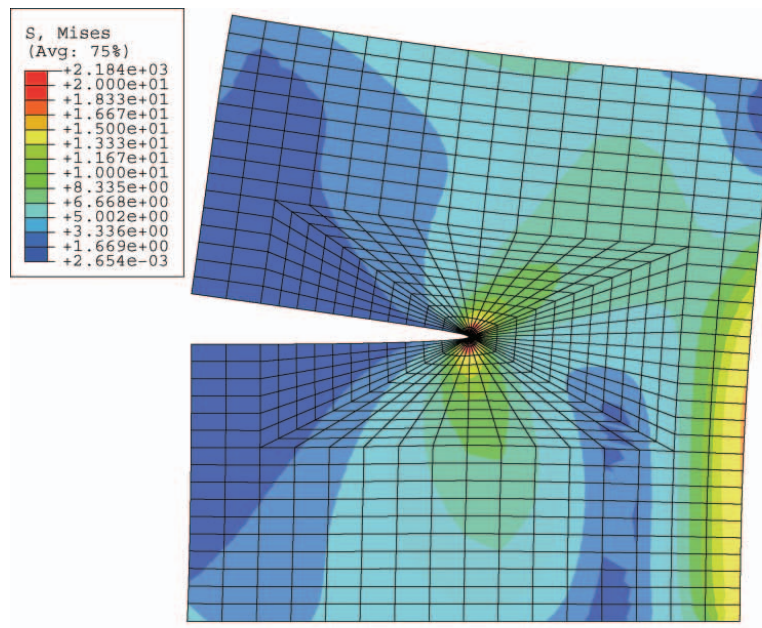

(a)
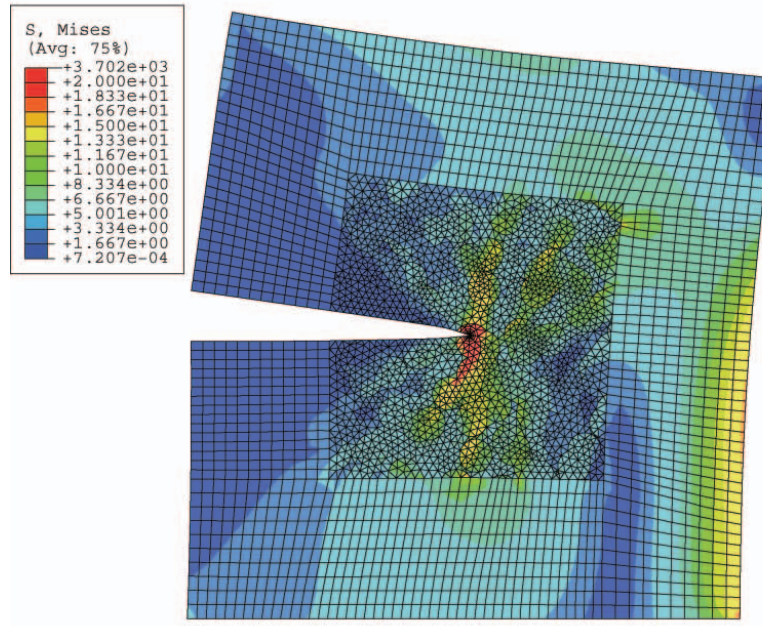

(c)
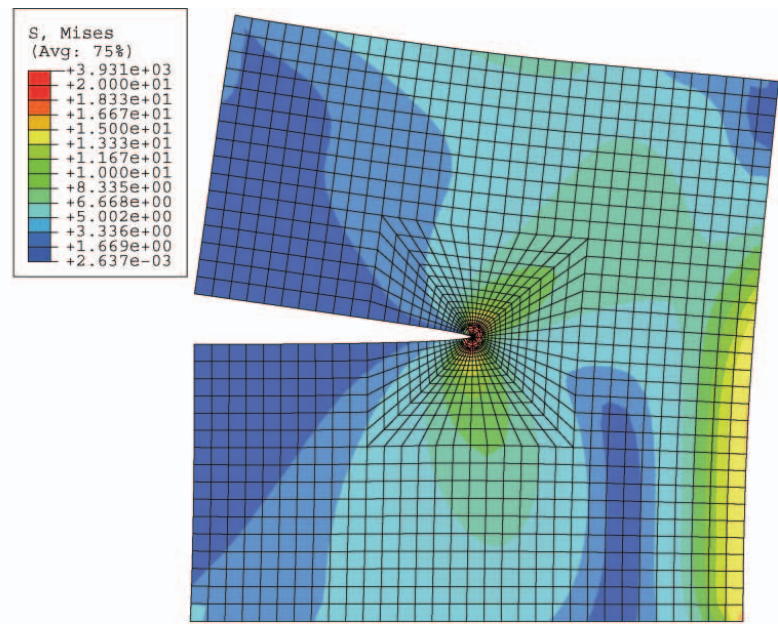

(b)
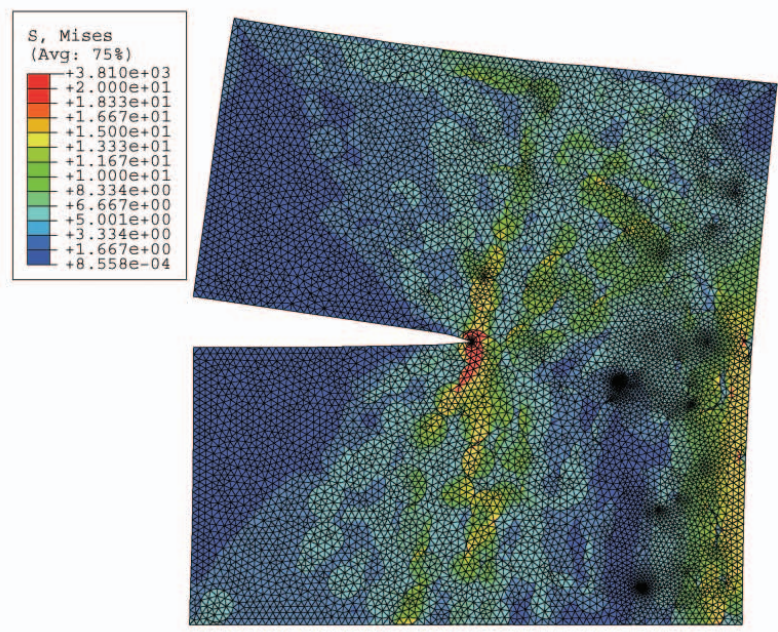

(d)

Figure 7. von Mises stress contours: (a) sequential; (b) invasive; (c) concurrent; (d) micro-scale.

means of SIFs than any other multi-scale models. The superior accuracy of the concurrent model extends to evaluating the standard deviations of SIFs, which are significantly under-predicted by all other multi-scale models except the invasive model, which predicts the standard deviation of $K_{I}$

Table 2. Statistics of SIFs by various models.

\begin{tabular}{lcclll}
\hline & \multicolumn{2}{c}{$K_{I}, \mathrm{MPa} \sqrt{ } \mathrm{m}$} & & \multicolumn{2}{c}{$K_{I I}, \mathrm{MPa} \sqrt{ } \mathrm{m}$} \\
\cline { 2 - 3 } Model & Mean & St. Dev. & & Mean & St. Dev. \\
\hline Sequential & 28.604 & 1.609 & & 2.77 & 0.263 \\
Invasive & 29.887 & 13.28 & 2.487 & 0.939 \\
Concurrent $(\kappa=0.25)$ & 30.095 & 13.083 & 2.2 & 2.25 \\
Concurrent $(\kappa=0.5)$ & 30.961 & 13.469 & 2.232 & 2.306 \\
Micro-scale & 32.213 & 13.998 & & 2.191 & 2.391 \\
\hline
\end{tabular}

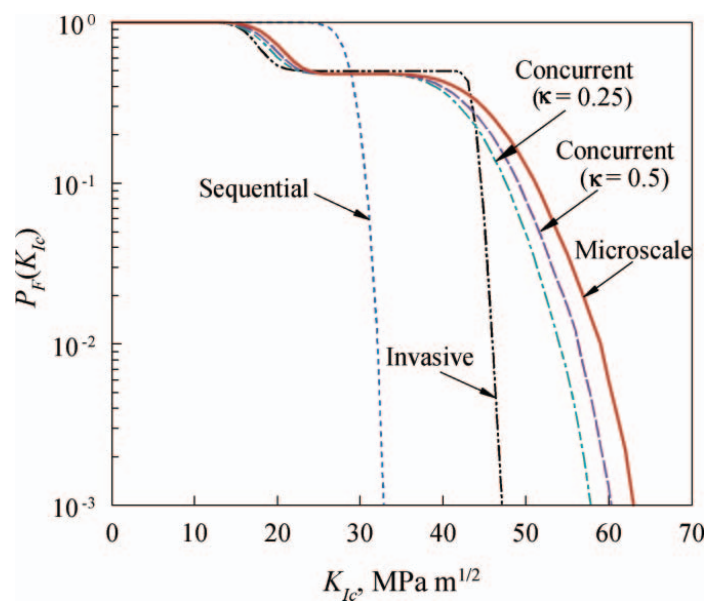

Figure 8. Conditional probability of fracture initiation. 
accurately. The accuracy of the concurrent models slightly improves when $\kappa$ is larger, e.g. $\kappa=0.5$, as expected.

A more meaningful stochastic response is the fracture reliability. Based on the maximum circumferential theory, Figure 8 plots how $P_{F}\left(K_{\text {Ic }}\right)$, the conditional probability of fracture initiation, varies as a function of the fracture toughness $K_{I c}$ of the FGM by the multi-scale models, including the reference solution from the micro-scale model. The results indicate that the sequential model not only fails to capture the effect of material property at the crack tip, but also gives misleading information about the failure probability curve. For $P_{F}\left(K_{I c}\right)<0.5$, the sequential model significantly under-predicts the probability of fracture initiation and is, therefore, unconservative. The invasive model gives a better estimate of the failure probability curve than those two aforementioned models, but still under-predicts failure probabilities less than 0.5 . If further accuracy is desired, the concurrent model, which provides more accurate prediction of the probability of fracture initiation than all other multi-scale models, can be employed. The accuracy of the concurrent model improves when $\kappa$ is increased from 0.25 to 0.5 , as expected.

For simulating crack-propagation, two deterministic samples of the FGM specimen in conjunction with the concurrent model $(\kappa=0.5)$ and the micro-scale model were examined. The deterministic boundary conditions are the

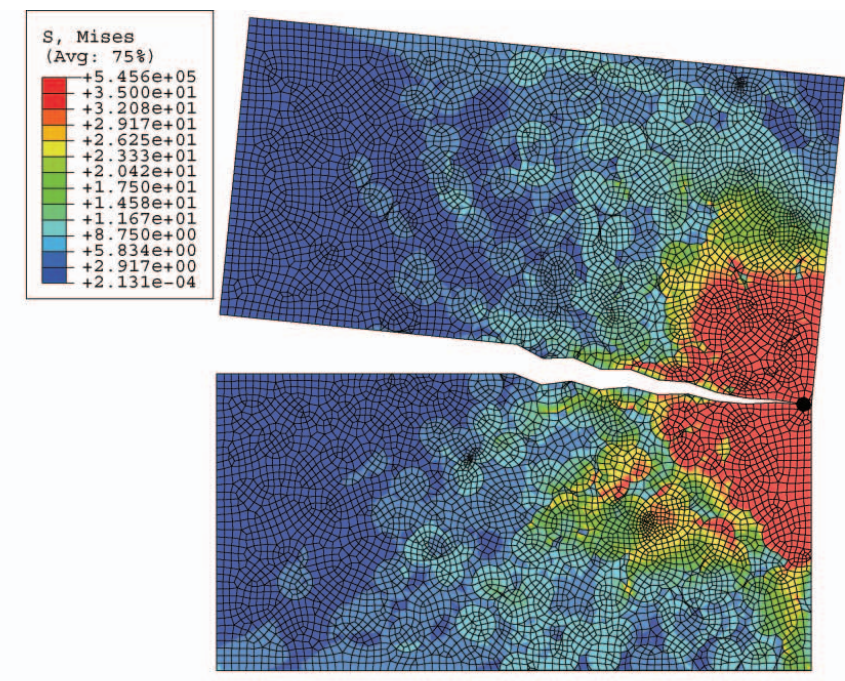

(a)

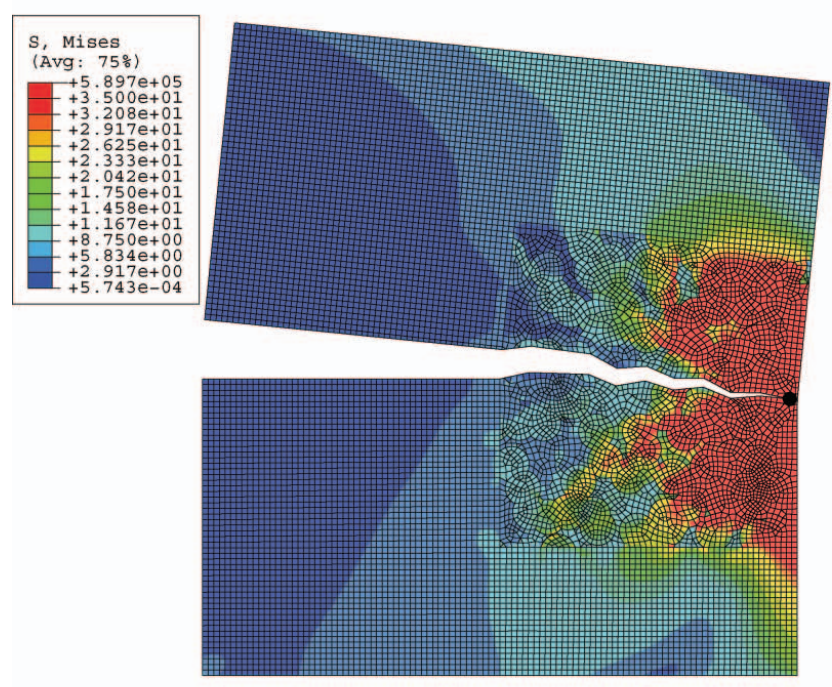

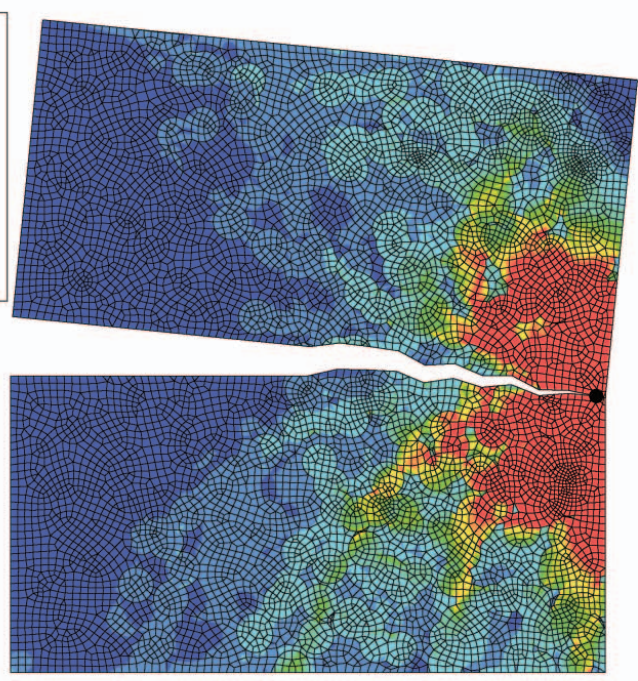

(b)

Figure 9. Simulation of crack propagation by concurrent multi-scale and micro-scale models: (a) sample 1; (b) sample 2. 
Table 3. CPU times by various models.

\begin{tabular}{lcc}
\hline Model & $\begin{array}{c}\text { Relative CPU } \\
\text { (single sample) }\end{array}$ & $\begin{array}{c}\text { Relative CPU } \\
\text { (full stochastic) }\end{array}$ \\
\hline Sequential & 0.18 & 0.02 \\
Invasive & 0.193 & 0.044 \\
Concurrent $(\kappa=0.25)$ & 0.255 & 0.255 \\
Concurrent $(\kappa=0.5)$ & 0.419 & 0.419 \\
Micro-scale & 1 & 1 \\
\hline
\end{tabular}

same as before. For both samples, the crack trajectories were determined based on the maximum circumferential stress theory. Figure 9a shows the crack propagation paths in one sample generated from the concurrent multi-scale and micro-scale models, where the initial propagation direction is downward. For the other sample, where the initial propagation direction is upward, the propagation paths are presented in Figure 9b, again obtained from both models. It is observed that the concurrent multi-scale model $(\kappa=0.5)$ gives almost identical crack propagation paths compared with the micro-scale model.

\subsubsection{Computational effort}

Table 3 lists the relative computational effort by the different multi-scale and micro-scale models examined in this work. The relative CPU time associated with a particular model is defined as the absolute CPU time required by that model divided by the absolute CPU time required by the micro-scale model. The second column of Table 3 displays the relative CPU time required for a single sample calculation that involves one deterministic finite-element analysis including all pre-processing efforts. The third column of Table 3 describes the relative CPU time for a complete stochastic fracture analysis that leads to the conditional failure probability curve in Figure 8. In both analyses, the micro-scale model is not only the most expensive, but also the most accurate. The sequential model is the most computationally inexpensive model, but they do not produce acceptable results. The invasive model, which gives mixed results, exhibits a similar computational efficiency to that of the sequential model. The concurrent multi-scale model, which produces sufficiently accurate results, is more expensive than other multi-scale models, but is still less expensive than the micro-scale model.

\section{Conclusions and outlook}

New stochastic models were developed for generating microstructures and multi-scale fracture analysis of random heterogeneous materials. The microstructure model is based on a level-cut inhomogeneous, filtered Poisson field comprising a sum of deterministic kernel functions that are scaled by random variables and centred at Poisson points. The fracture model involves stochastic descrip- tion of the particle volume fraction and locations and constituent material properties; a two-scale algorithm including micro-scale and macro-scale analyses for determining crack-driving forces; and dimensional decomposition or Monte Carlo simulation for reliability analysis. Numerical results demonstrate that the random field model is capable of producing a wide variety of two- and threedimensional microstructures containing particles of various sizes, shapes, densities, gradations and orientations. The results from an edge-cracked, functionally graded specimen under a mixed-mode deformation indicate that (1) the simpler multi-scale models, the sequential and the invasive models are the most computationally inexpensive models available, but they do not produce acceptable probabilistic characteristics of stress-intensity factors or accurate probability of fracture initiation; and (2) the concurrent model is sufficiently accurate, gives probabilistic solutions very close to those generated from the micro-scale model and can reduce the computational effort of the latter model by more than a factor of two. Finally, a limited yet demonstrative study on crack-propagation simulation indicates that the concurrent multi-scale model can also predict crack trajectory accurately.

The stochastic modelling of multi-scale fracture presented in this paper has the potential to fundamentally change the way advanced materials in high-technology applications, including the maritime industry, can be applied in the future. The ability to model microstructure and simulate the structure-property relationship of advanced composites should pave the way for a new design paradigm. The associated numerical tools developed are expected to reduce the need for costly experimentation, create new information not evident through experimentation alone and lead to designing lighter, stronger, tougher composites.

\section{Acknowledgements}

The author would like to acknowledge financial support from the US National Science Foundation under Grant No. CMS-0409463. The author thanks A. Chakraborty for conducting fracture analyses of FGMs

\section{References}

ABAQUS. 2006. User's guide and theoretical manual. Ver. 6.6. Providence (RI): Simulia-Dassault Systèms.

Anderson T. 2005. Fracture mechanics: fundamentals and applications. 3rd ed. Boca Raton (FL): CRC Press.

Chakraborty A, Rahman S. 2008. Stochastic multiscale fracture of functionally graded materials. Eng Fract Mech. 75:20622086.

Dutta PK. 1994. Low-temperature compressive strength of glassfiber-reinforced polymer composites. J Offshore Mech Arct. 116:167-172.

Einstein A. 1906. Eine neue bestimmung der moleküldimensionen. Ann Phys. 19:289-306.

Ferrante F, Graham-Brady L. 2005. Stochastic simulation of nonGaussian/non-stationary properties in a functionally graded plate. Comput Method Appl M. 194:1675-1692. 
Gillespie, JW. 1991. Damage tolerance of composite structures: the role of interlaminar fracture mechanics. J Offshore Mech Arct. 113:247-252.

Grigoriu M. 2003. Random field models for two-phase microstructures. J Appl Phys. 94:3762-3770.

Maxwell J. 1873. Treatise on electricity and magnetism. Oxford (UK): Clarendon Press.

Mura T. 1991. Micromechanics of defects in solids. 2nd rev. ed. Dordrecht (The Netherlands): Kluwer Academic Publishers.

Quintanilla J, Torquato S. 1997. Microstructure functions for a model of statistically inhomogeneous random media. Phys Rev E. 55(2):1558-1565.

Rahman S. 2008. A random field model for generating synthetic microstructures of functionally graded materials. Int J Numer Meth Eng. 76:972-993.
Rahman S, Chakraborty A. 2007. A stochastic micromechanical model for elastic properties of functionally graded materials. Mech Mater. 39:548-563.

Rao B, Rahman S. 2003. Meshfree analysis of cracks in isotropic functionally graded materials. Eng Fract Mech. 70(1):1-27.

Rayleigh L. 1892. On the influence of obstacles arranged in a rectangular order upon the properties of medium. Phil Mag. 34:481-502.

Roberts A. 1997. Statistical reconstruction of three-dimensional porous media from two-dimensional images. Phys Rev E. 56(3):3203-3212.

Torquato S. 2002. Random heterogeneous materials: microstructures and macroscopic properties. New York (NY): Springer.

Xu H, Rahman S. 2005. Decomposition methods for structural reliability analysis. Probabilist Eng Mech. 20:239-250. 